\title{
Risk of Pneumonia Associated with Inhaled Corticosteroid in Patients with Chronic Obstructive Pulmonary Disease: A Korean Population-Based Study
}

This article was published in the following Dove Press journal:

International Journal of Chronic Obstructive Pulmonary Disease

\author{
Ji-Ho Lee (D) ${ }^{1, *}$ \\ You Hyun Park $\mathbb{D}^{2, *}$ \\ Dae Ryong Kang ${ }^{3}$ \\ Seok Jeong Lee $\mathbb{D}^{\prime}$ \\ Myoung Kyu Lee $\mathbb{D}^{\prime}$ \\ Sang-Ha Kim (D)' \\ Suk Joong Yong' \\ Won-Yeon Lee (D) \\ 'Department of Internal Medicine, Yonsei \\ University Wonju College of Medicine, \\ Wonju, Korea; ${ }^{2}$ Department of \\ Biostatistics, Yonsei University, Seoul, \\ Korea; ${ }^{3}$ Department of Precision \\ Medicine \& Biostatistics, Yonsei \\ University Wonju College of Medicine, \\ Wonju, Korea
}

*These authors contributed equally to this work
Correspondence: Won-Yeon Lee Department of Internal Medicine, Yonsei University Wonju College of Medicine, 20 Ilsan-Ro, Wonju 26426, Korea

Tel +82-33-74I-0926

Fax +82-33-74I-0928

Email wonylee@yonsei.ac.kr
Introduction: Inhaled corticosteroids (ICSs) are recommended for patients with frequent exacerbation of chronic obstructive pulmonary disease (COPD). However, accumulating evidence has indicated the risk of pneumonia from the use of ICS. This study aimed to investigate the association between ICS and pneumonia in the real-world clinical setting.

Methods: A retrospective cohort study was performed using nationwide population data from the Korea National Health Insurance Service. Subjects who had a new diagnosis of COPD and who received inhaled bronchodilators without a diagnosis of pneumonia before the initiation of bronchodilators were identified. Subjects were followed up until their first diagnosis of pneumonia. The risk of pneumonia in ICS users was compared to that in nonICS users.

Results: A total of 87,594 subjects were identified and 1:1 matched to 22,161 ICS users and non-ICS users. More ICS users were diagnosed with pneumonia compared to non-ICS users (33.73\% versus $24.51 \%, P<0.0001$ ). The incidence rate per 100,000 person-years was 8904.98 for ICS users and 6206.79 for non-ICS users. The hazard ratio (HR) of pneumonia for ICS users was 1.62 (95\% CI 1.54-1.70). The HR of subjects prescribed with the lowest ICS cumulative dose was 1.35 (1.27-1.43). The HR increased to 1.51 (1.42-1.60), 1.96 (1.85-2.09), and 2.03 (1.89-2.18) as the cumulative dose increased. Pneumonia was strongly associated with fluticasone propionate $(1.79(1.70-1.89))$ and fluticasone furoate $(1.80(1.61-2.01))$ use, compared to the use of other types of ICS.

Conclusion: ICS increases the risk of pneumonia in patients with COPD. Hence, ICS should be carefully prescribed in patients with risk factors for pneumonia while considering the cumulative doses and subtypes of ICS.

Keywords: inhaled corticosteroid, chronic obstructive pulmonary disease, pneumonia, fluticasone

\section{Introduction}

Chronic obstructive pulmonary disease (COPD) is a chronic inflammatory lung disease characterized by irreversible airflow limitations. The principal treatment strategy includes the use of long-acting inhaled bronchodilators, such as long-acting muscarinic antagonists (LAMA) and long-acting $\beta_{2}$ agonists (LABA). ${ }^{1}$ They can improve respiratory symptoms, lung function and physical activity, and notably decrease the exacerbation rate. ${ }^{2}$ The use of inhaled corticosteroids (ICSs) is another 
therapeutic strategy for COPD. However, the role of ICS in the treatment of COPD remains controversial. ${ }^{3,4}$ Therefore, ICS is recommended for specific target populations in the current COPD guidelines. ${ }^{5}$

Although ICS has been beneficial in terms of reducing the rate of acute exacerbation, it entails various adverse effects. ${ }^{6}$ Respiratory adverse effects include pneumonia, tuberculosis, and non-tuberculous mycobacterial infection. Systemic adverse effects include diabetes, bone fractures, cataracts, and adrenal insufficiency. ${ }^{7}$ Among these, pneumonia can cause potential damage to patients with COPD because it is closely linked with COPD exacerbation. ${ }^{8}$ The association of ICS use with pneumonia has been reported in many studies including randomized controlled trials (RCTs) and meta-analyses. ${ }^{9}$ However, there are still inconsistent results regarding which types of ICS are not associated with the likelihood of developing pneumonia. ${ }^{10}$

Real-life observational studies include a more heterogeneous population with broader inclusion criteria that could be excluded in RCTs. ${ }^{11}$ They can support or strengthen the results of previous RCTs with narrow inclusion criteria. However, there is limited evidence of an association between ICS use and pneumonia in observational studies. Studies with long-term observational periods of more than 10 years are scarce, and almost all data are obtained from Western countries or their local communities, not from a nationwide population-based cohort. In addition, the risk of pneumonia for cumulative doses of ICS and ICS types other than fluticasone propionate and budesonide has not been addressed. This study aimed to determine whether the use of ICS elevates the risk of pneumonia in patients with COPD in a nationwide population cohort with an observation period of 13 years.

\section{Methods}

\section{Data Source}

In our retrospective cohort study, data from the Korea National Health Insurance Service (NHIS) were used. The NHIS is a unique health insurance program launched in 2002 by the government, covering approximately $97 \%$ of the Korean population. The remaining 3\% of the population is insured by the Medical Assistance Program. Healthcare providers, including those from clinics, hospitals, and pharmacies in Korea, submit claims of their healthcare services to the NHIS electronically; these are then reimbursed. The claims data of the NHIS include healthcare utilization information for both inpatients and outpatients, such as patient demographics, diagnoses, procedures, surgical histories, and prescription drugs, which are provided for research purposes. The International Classification of Disease and Related Health Problems, 10th revision (ICD-10), was adopted in the NHIS to classify diseases according to diagnostic codes (Supplementary Table S1). Data on the diagnostic codes, diagnostic procedures, and prescription information including product names, dosages, prescription dates, and durations were collected.

\section{Study Subjects}

Among the entire nationwide population data, subjects with a diagnostic code of COPD (J42- J44, except J430) at least two times between January 01, 2005, and December 31, 2018, were included, and subjects with a diagnostic code of lung malignancy at least one time during the same period were excluded. In addition, subjects who did not undergo pulmonary function tests 1 year before or after the COPD diagnosis were excluded. Then, data were randomly $50 \%$ sampled by a revised NHIS policy for medical research related to medications. The subjects with COPD $(n=87,594)$ were included from the claims data if the following criteria were met: 1) age $\geq 40$ years; 2) new COPD diagnostic code since January 01, 2005 ; 3) prescription of at least one type of inhaled bronchodilator such as LAMA, LABA, ICS, or shortacting $\beta_{2}$ agonist (SABA), after the COPD diagnosis; 4) no prescription with different components of ICS simultaneously; 5) no diagnosis of pneumonia before the index date; 6) no death before the index date; and 7) sufficient medication records. Study subjects were followed up until their initial diagnosis of pneumonia, their death, or to end of the follow-up period, whichever came first.

ICS users were defined as having prescribed ICS for at least 1 month, while non-ICS users were defined as those not prescribed ICS ever during the study period. Index date was calculated as the day of the first prescription of LAMA, LABA, and/or SABA for non-ICS users, and the day of the first prescription of ICS for ICS users. The bronchodilators prescribed during the 12 months after the index date were classified as SABA, LAMA, LABA, and LAMA + LABA combinations. Comorbidities were identified if at least two diagnostic codes were recorded during the study period, from the initial diagnosis of COPD to the final follow-up date. Asthma, interstitial lung disease, diabetes, hypertension, heart failure, chronic kidney disease, and chronic liver disease were considered as comorbidities. Severe exacerbation of COPD was defined as 
a history of admission within 12 months prior to the index date where the main diagnosis was COPD. The proportion of subjects who received oral corticosteroids (OCSs) and OCS prescriptions day in the 12 months prior to the index date was analyzed. The intervals from the subjects' COPD diagnosis to the index date were also calculated. This study was approved by the Institutional Review Board of Wonju Severance Christian Hospital (CR318361). The requirement for informed consent was waived for the study as it retrospectively analyzed anonymous claim data.

\section{Study Outcomes}

The primary endpoint was the difference in the development of pneumonia between ICS users and non-ICS users. The secondary endpoint was the difference in the development of pneumonia derived from the subgroup analysis of ICS users: 1) cumulative dose of ICS, 2) daily dose of ICS, and 3) type of ICS. Cases of pneumonia were defined as cases with an initial claim date under the diagnostic code of pneumonia (J10-J18) in the time since the index date, regardless of whether they were claimed at inpatient or outpatient clinics. ${ }^{12}$ The cumulative ICS dose was the summation of all prescribed doses of ICS, which was divided into four groups of quartiles according to $25 \%$, $50 \%$, and $75 \%$ of the population. The daily dose of ICS was calculated by dividing the total prescribed doses of ICS by the prescription period. The dosages of the various types of ICSs were converted into the equivalent doses of fluticasone propionate. Fluticasone propionate $(50 \mu \mathrm{g})$ was equivalent to beclomethasone $(100 \mu \mathrm{g})$, beclomethasone HFA $(50 \mu \mathrm{g})$, budesonide ( $80 \mu \mathrm{g})$, ciclesonide $(32 \mu \mathrm{g})$, and fluticasone furoate $(10 \mu \mathrm{g}) .{ }^{13}$ Daily doses of ICS were classified into low (1-499 $\mu \mathrm{g})$, medium (500-999 $\mu \mathrm{g})$ and high doses $(\geq 1000 \mu \mathrm{g})$ in reference to fluticasone propionate.

\section{Statistical Analysis}

ICS users and non-ICS users were chosen by applying propensity score matching to decrease selection bias. A 1:1 matching between ICS users and non-ICS users was performed using a logistic regression that included variables such as age, sex, comorbidities, history of COPD exacerbation, presence of OCS prescription, duration of OCS prescription, and interval period from COPD diagnosis to the index date. Baseline clinical characteristics of the ICS users and non-ICS users were compared using Student's $t$-test for continuous variables and the Chisquare test for categorical variables. Survival analysis over time was analyzed using Kaplan-Meier curves with a Log rank test. The types of ICSs were not included in the survival analysis because the durations of the prescriptions of some ICSs such as beclomethasone, ciclesonide, and fluticasone furoate were insufficient. Cox proportional hazards analyses were conducted to estimate the hazard ratio (HR) and 95\% confidence interval (CI) for the association between the prescription of ICS and the development of pneumonia. All statistical analyses were performed using SAS 9.4 (SAS Institute Inc., Cary, NC, USA). A $P$-value less than 0.05 was considered statistically significant.

\section{Results}

\section{Demographics of the Study Subjects}

The number of subjects diagnosed with COPD without lung cancer between 2005 and 2018 and who were receiving pulmonary function tests before or after 1 year since their COPD diagnosis was $1,545,246$. The exclusion criteria were applied to half of the subjects through $50 \%$ sampling. As a result, 87,594 subjects with COPD were identified (59,694 ICS users and 27,900 non-ICS users). Both groups were finally matched to 22,161 ICS users and 22,161 non-ICS users (Figure 1).

Age distribution, sex, and comorbidities did not differ between ICS users and non-ICS users. Mean age was 65.4 \pm 11.0 years for ICS users and $65.4 \pm 11.0$ years for nonICS users $(P=0.9281)$. The proportion of male subjects was $72.40 \%$ for ICS users and $71.91 \%$ for non-ICS users $(P=0.2435)$. The history of COPD exacerbation and the proportion of OCS prescriptions 12 months prior to the index date were also comparable between the two groups. OCS prescription duration was $8.44 \pm 13.56$ days for ICS users, which was longer than the $7.79 \pm 13.93$ days for non-ICS users $(P<0.0001)$. There was a difference in the use of bronchodilators in addition to ICS use. SABA and LAMA were prescribed more frequently for non-ICS users compared to ICS users, while LABA was more frequently used for ICS users than for non-ICS users (Table 1).

Fluticasone propionate was the most frequently prescribed ICS $(51.60 \%)$, followed by budesonide $(22.80 \%)$, fluticasone furoate $(11.37 \%)$, beclomethasone $(9.73 \%)$, and ciclesonide $(4.50 \%)$. The mean cumulative dose of ICSs was $132,315.7 \pm 338,241.0 \mu \mathrm{g}$. The cumulative doses of ICS were divided into four quartiles: 1-15,000 $\mu \mathrm{g}$ (Q1), 15,000-30,720 $\mu \mathrm{g}$ (Q2), 30,721-107,160 $\mu \mathrm{g}$ (Q3), and >107,610 $\mu \mathrm{g}$ (Q4) according to the proportion 


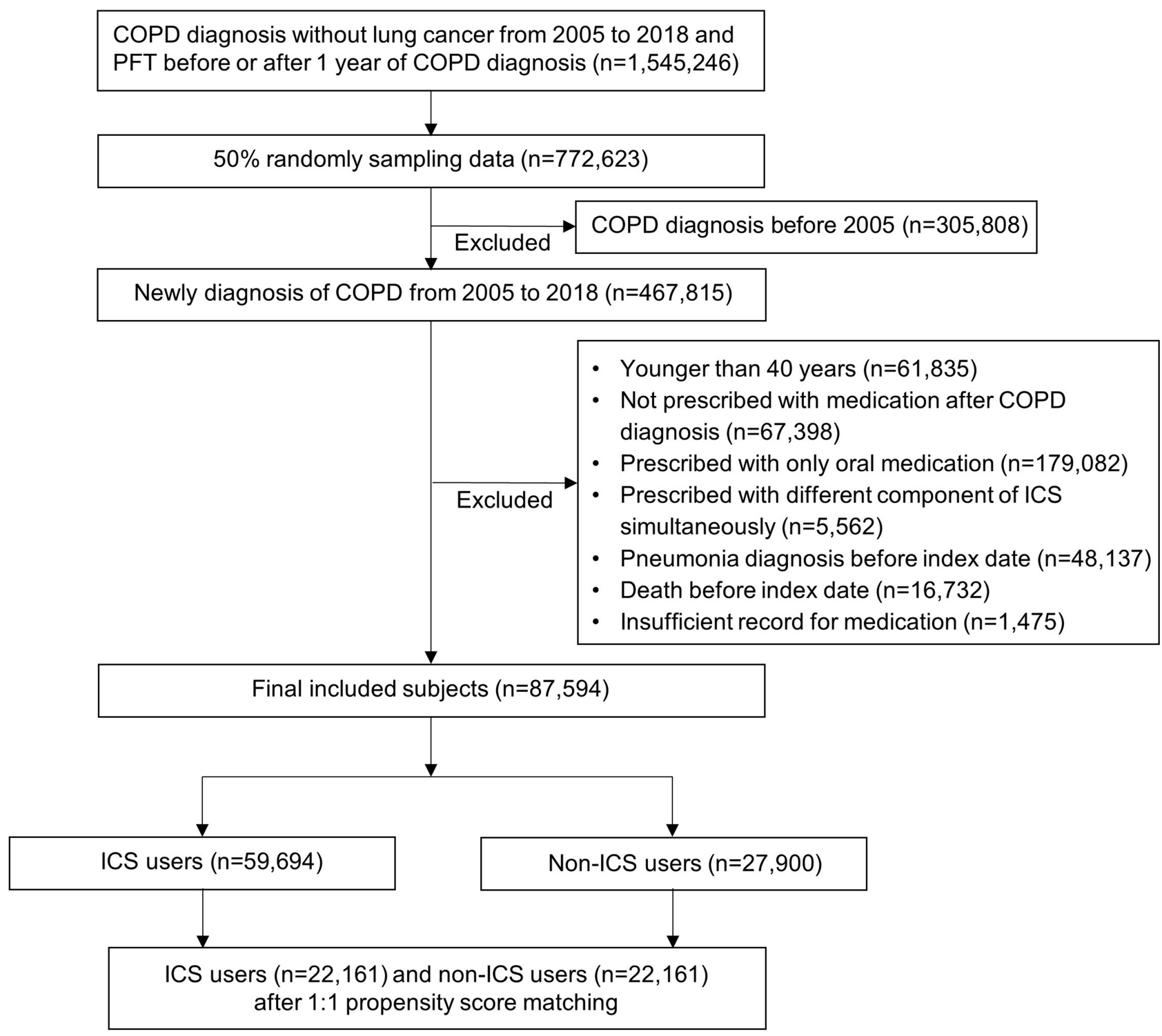

Figure I Patient selection flowchart.

of subjects. For the daily doses of ICS, $60.81 \%$ and $33.08 \%$ were classified as low and medium doses, respectively, while $6.11 \%$ were classified as on high doses (Table 2).

\section{Incidence of Pneumonia in ICS Users}

For ICS users, 7473 (33.73\%) subjects were identified as having pneumonia during the study period, which was larger than the $5432(24.51 \%)$ group of non-ICS users $(P<0.0001)$. The incidence rate per 100,000 person-years was 8904.98 for ICS users and 6206.79 for non-ICS users. The incidence of pneumonia increased with an increase in the cumulative ICS dose. Incidence rate was 7407.53 in Q1, 7502.87 in Q2, 9376.11 in Q3, and 11,096.63 in Q4.
However, the incidence of pneumonia did not correlate with the daily ICS dose. The incidence rate was 8771.55 for low doses, 9126.64 for medium doses, and 8836.10 for high doses. The incidence of pneumonia varied according to the type of ICS. The incidence rate was 9434.95 for fluticasone propionate, 7614.98 for budesonide, 8388.57 for beclomethasone, 7252.61 for ciclesonide, and 12,278.90 for fluticasone furoate (Table 3 ). The cumulative proportion of patients who had a diagnosis of pneumonia over time was analyzed. In ICS users, pneumonia occurred more frequently than in non-ICS users (Figure 2A). Pneumonia occurred gradually over time rather than abruptly at a specific peak time. In addition, pneumonia occurred more frequently in those receiving higher cumulative 
Table I Baseline Characteristics of the Study Subjects

\begin{tabular}{|c|c|c|c|c|c|}
\hline & \multicolumn{2}{|c|}{$\begin{array}{l}\text { ICS Users } \\
(n=22,161)\end{array}$} & \multicolumn{2}{|c|}{$\begin{array}{l}\text { Non-ICS } \\
\text { Users } \\
(n=22,161)\end{array}$} & \multirow[t]{2}{*}{$P$ value } \\
\hline & $\mathbf{n}$ & $\%$ & $\mathbf{n}$ & $\%$ & \\
\hline \multicolumn{6}{|l|}{ Age } \\
\hline Mean (SD) & \multicolumn{2}{|c|}{$65.4(11.0)$} & \multicolumn{2}{|c|}{$65.4(11.0)$} & 0.9281 \\
\hline $40-49$ & 1968 & 8.88 & 2021 & 9.12 & 0.4082 \\
\hline $50-59$ & 4590 & 20.71 & 4742 & 21.40 & \\
\hline $60-69$ & 7106 & 32.07 & 7059 & 31.85 & \\
\hline 70-79 & 6329 & 28.56 & 6208 & 28.01 & \\
\hline$\geq 80$ & 2168 & 9.78 & 2131 & 9.62 & \\
\hline \multicolumn{6}{|l|}{ Sex } \\
\hline Male & 16,044 & 72.40 & 15,935 & 71.91 & 0.2435 \\
\hline Female & 6117 & 27.60 & 6226 & 28.09 & \\
\hline \multicolumn{6}{|l|}{ Comorbidity } \\
\hline Asthma & 10,926 & 49.30 & 11,318 & 51.07 & 0.0060 \\
\hline $\begin{array}{l}\text { Interstitial lung } \\
\text { disease }\end{array}$ & 325 & 1.47 & 344 & 1.55 & 0.3115 \\
\hline Diabetes & 5623 & 25.37 & 5665 & 25.56 & 0.5779 \\
\hline Hypertension & 10,105 & 45.60 & 10,242 & 46.22 & 0.0859 \\
\hline Heart failure & 3746 & 16.90 & 3792 & 17.11 & 0.5436 \\
\hline $\begin{array}{l}\text { Chronic kidney } \\
\text { disease }\end{array}$ & 839 & 3.79 & 881 & 3.98 & 0.3247 \\
\hline Chronic liver disease & 6609 & 29.82 & 6716 & 30.31 & 0.1557 \\
\hline \multicolumn{6}{|l|}{$\mathrm{CCl}$} \\
\hline Mean (SD) & \multicolumn{2}{|c|}{$2.78(1.94)$} & \multicolumn{2}{|c|}{$2.83(1.98)$} & 0.0375 \\
\hline$<2$ & 12,195 & 55.03 & 0.0050 & 53.96 & 0.0050 \\
\hline$\geq 2$ & 9966 & 44.97 & 10,203 & 46.04 & \\
\hline \multicolumn{6}{|l|}{ Bronchodilator } \\
\hline SABA & $126 \mid$ & 5.69 & 6166 & 27.82 & $<0.0001$ \\
\hline LAMA & 119 & 0.54 & 8761 & 39.53 & \\
\hline LABA & 15,054 & 67.93 & 1037 & 4.68 & \\
\hline LAMA + LABA & 5727 & 25.84 & 6197 & 27.96 & \\
\hline \multicolumn{6}{|c|}{ Severe exacerbation of COPD } \\
\hline Yes & 990 & 4.47 & 1045 & 4.72 & 0.1880 \\
\hline No & 21,171 & 95.53 & 16,729 & 95.28 & \\
\hline
\end{tabular}

(Continued)
Table I (Continued).

\begin{tabular}{|c|c|c|c|c|c|}
\hline & \multicolumn{2}{|c|}{$\begin{array}{l}\text { ICS Users } \\
(n=22,161)\end{array}$} & \multicolumn{2}{|c|}{$\begin{array}{l}\text { Non-ICS } \\
\text { Users } \\
(n=22,161)\end{array}$} & \multirow[t]{2}{*}{$P$ value } \\
\hline & $\mathbf{n}$ & $\%$ & $\mathbf{n}$ & $\%$ & \\
\hline \multicolumn{6}{|c|}{ OCS prescription } \\
\hline Yes & 17,186 & 77.55 & 17,322 & 78.16 & 0.1336 \\
\hline No & 4975 & 22.45 & 4839 & 21.84 & \\
\hline \multicolumn{6}{|c|}{ OCS prescription day } \\
\hline Mean (SD) & \multicolumn{2}{|c|}{$8.44(13.56)$} & \multicolumn{2}{|c|}{$7.79(13.93)$} & $<0.0001$ \\
\hline \multicolumn{6}{|c|}{ Interval from COPD diagnosis to index date } \\
\hline Mean (SD) & \multicolumn{2}{|c|}{$315.3(7 \mid 1.7)$} & \multicolumn{2}{|c|}{$344.6(801.5)$} & $<0.0001$ \\
\hline
\end{tabular}

Abbreviations: ICS, inhaled corticosteroid; LAMA, long-acting muscarinic antagonist; LABA, long-acting $\beta_{2}$ agonist.

doses of ICS (Figure 2B). The daily dose of ICS did not influence the occurrence of pneumonia (Figure 2C).

\section{Risk of Pneumonia in ICS Users}

The risk of pneumonia in ICS users and their subgroup was compared to that of non-ICS users. HR for the development of pneumonia in ICS users was $1.62(95 \%$ CI $1.54-1.70)(P<$ 0.0001). The HR of Q1 subjects prescribed with the lowest ICS cumulative dose was $1.35(1.27-1.43)(P<0.0001)$. HR increased to 1.51 (1.42-1.60) in Q2 subjects, 1.96 (1.85-2.09) in Q3 subjects, and 2.03 (1.89-2.18) in Q4 subjects prescribed with the highest ICS cumulative doses (all $P<0.0001$ ). The risk of pneumonia did not correlate with the daily ICS dose. HR was 1.59 (1.51-1.68) in low-dose ICS users, 1.67 (1.59-1.77) in medium-dose ICS users, and 1.55 (1.40-1.71) in high-dose ICS users (all $P<0.0001$ ). The risk of pneumonia varied according to the type of ICS. The HRs of fluticasone propionate and fluticasone furoate were $1.79(1.70-1.89)$ and 1.80 (1.61-2.01), respectively (all $P<0.0001$ ). However, HR of other types of ICS decreased to $1.44(1.35-1.54)$ in budesonide, $1.52(1.38-1.68)$ in beclomethasone, and 1.29 (1.14-1.45) in ciclesonide (all $P<0.0001$ ) (Figure 3 and Supplementary Table S2).

\section{Discussion}

In this study, the use of ICS was significantly associated with the development of pneumonia in COPD patients. The risk of pneumonia increased with an increase in the cumulative ICS cumulative dose, while it was not correlated with the daily dose of ICS. In addition, the risk of 
Table 2 Characteristics of Inhaled Corticosteroid Users

\begin{tabular}{|c|c|c|}
\hline & \multicolumn{2}{|l|}{ ICS User (Total 22,26I) } \\
\hline & $\mathbf{n}$ & $\%$ \\
\hline \multicolumn{3}{|l|}{ ICS cumulative dose } \\
\hline Mean (SD) & $|32,3| 5.7(338,24 \mid .0)$ & \\
\hline Medium (QI, Q3) & $30,720(15,000,107,160)$ & \\
\hline $\mathrm{I}-15,000 \mu \mathrm{g}$ & 5542 & 25.01 \\
\hline $15,00 \mathrm{I}-30,720 \mu \mathrm{g}$ & 5925 & 26.73 \\
\hline $30,721-107,160 \mu g$ & 5156 & 23.27 \\
\hline$>107,610 \mu \mathrm{g}$ & 5538 & 24.99 \\
\hline \multicolumn{3}{|l|}{ ICS dose } \\
\hline Low & 13,477 & 60.81 \\
\hline Medium & 7330 & 33.08 \\
\hline High & 1354 & 6.11 \\
\hline \multicolumn{3}{|l|}{ Type of ICS } \\
\hline Fluticasone propionate & 11,436 & 51.60 \\
\hline Budesonide & 5062 & 22.80 \\
\hline Beclomethasone & 2156 & 9.73 \\
\hline Ciclesonide & 997 & 4.50 \\
\hline Fluticasone furoate & 2520 & 11.37 \\
\hline
\end{tabular}

Abbreviation: ICS, inhaled corticosteroid.

pneumonia differed according to the types of ICS; fluticasone propionate and fluticasone furoate were highly associated with pneumonia compared to other ICS types.

The risk of pneumonia due to ICS use was first reported in the TORCH study. ${ }^{14}$ In that study, the relative risk of pneumonia in ICS users compared to non-ICS users was $1.52(1.32-1.76)$ and the incidence rate of pneumonia per 100,000 persons was 5200 for non-ICS users and 8800 for ICS users, which is similar to our study results. Subsequent RCTs and meta-analyses also raised concerns about the risk of pneumonia from the use of ICS in COPD. ${ }^{15,16}$ However, some RCTs reported that ICS use did not increase the risk of pneumonia. ${ }^{17-19}$ These contradictory results in clinical trials might be attributable to the differences in study protocols and populations, the severity of COPD, risk factors of pneumonia, and misdiagnosis of pneumonia. ${ }^{20}$

RCTs can demonstrate a direct causal relationship through strict inclusion criteria. However, approximately $90 \%$ of COPD patients do not meet the inclusion criteria of these clinical trials. ${ }^{11}$ However, observational studies
Table 3 Crude Incidence Rate of Pneumonia According to the ICS Type and Dose

\begin{tabular}{|c|c|c|c|}
\hline Variables & $\begin{array}{l}\text { Person } \\
\text { Year }\end{array}$ & $\begin{array}{l}\text { Pneumonia } \\
\text { Patients }\end{array}$ & $\begin{array}{l}\text { Incidence Rate } \\
\text { (per } 100,000)\end{array}$ \\
\hline Non-ICS user & $87,517.00$ & 5432 & 6206.79 \\
\hline ICS user & $83,919.32$ & 7473 & 8904.98 \\
\hline \multicolumn{4}{|c|}{ ICS cumulative dose $(\mu \mathrm{g})$} \\
\hline $1-15,000$ & $22,396.12$ & 1659 & 7407.53 \\
\hline $15,000-30,720$ & $18,486.25$ & 1387 & 7502.87 \\
\hline $30,721-107,160$ & $20,264.26$ & 1900 & 9376.11 \\
\hline$>107,610$ & $22,772.68$ & 2527 & $11,096.63$ \\
\hline \multicolumn{4}{|l|}{ ICS dose } \\
\hline Low & $48,189.90$ & 4227 & 8771.55 \\
\hline Medium & $30,602.72$ & 2793 & 9126.64 \\
\hline High & 5126.70 & 453 & 8836.10 \\
\hline \multicolumn{4}{|l|}{ Type of ICS } \\
\hline $\begin{array}{l}\text { Fluticasone } \\
\text { propionate }\end{array}$ & $49,698.20$ & 4689 & 9434.95 \\
\hline Budesonide & $21,076.88$ & 1605 & $76 \mid 4.98$ \\
\hline Beclomethasone & 6139.31 & 515 & 8388.57 \\
\hline Ciclesonide & 3902.04 & 283 & 7252.61 \\
\hline $\begin{array}{l}\text { Fluticasone } \\
\text { furoate }\end{array}$ & 3102.88 & 381 & $12,278.90$ \\
\hline
\end{tabular}

Abbreviation: ICS, inhaled corticosteroid.

have a wide spectrum of inclusion criteria and can represent the actual population, including primary care settings. Previous observational studies using the health administrative databases of local communities or nations also showed an elevated risk of pneumonia from the use of ICSs in COPD. ${ }^{21-26}$ The relative risk of pneumonia in ICS users was 1.11-1.70. The present study also showed an increased risk of pneumonia in this patient population. Although additional evidence with rigorous criteria is necessary, the association between the risk of pneumonia and ICS use has been found in most observational studies, consolidating the positive results seen in the RCTs and meta-analyses.

The HR of 1.62 in the present study ranges within the risk seen in previous observational studies $(1.11-1.70){ }^{21-26}$ However, this figure is relatively higher than that in other studies. The reason for the higher risk of pneumonia might be attributable to the characteristics of Korean patients with COPD as well as the study duration. Low body mass index 

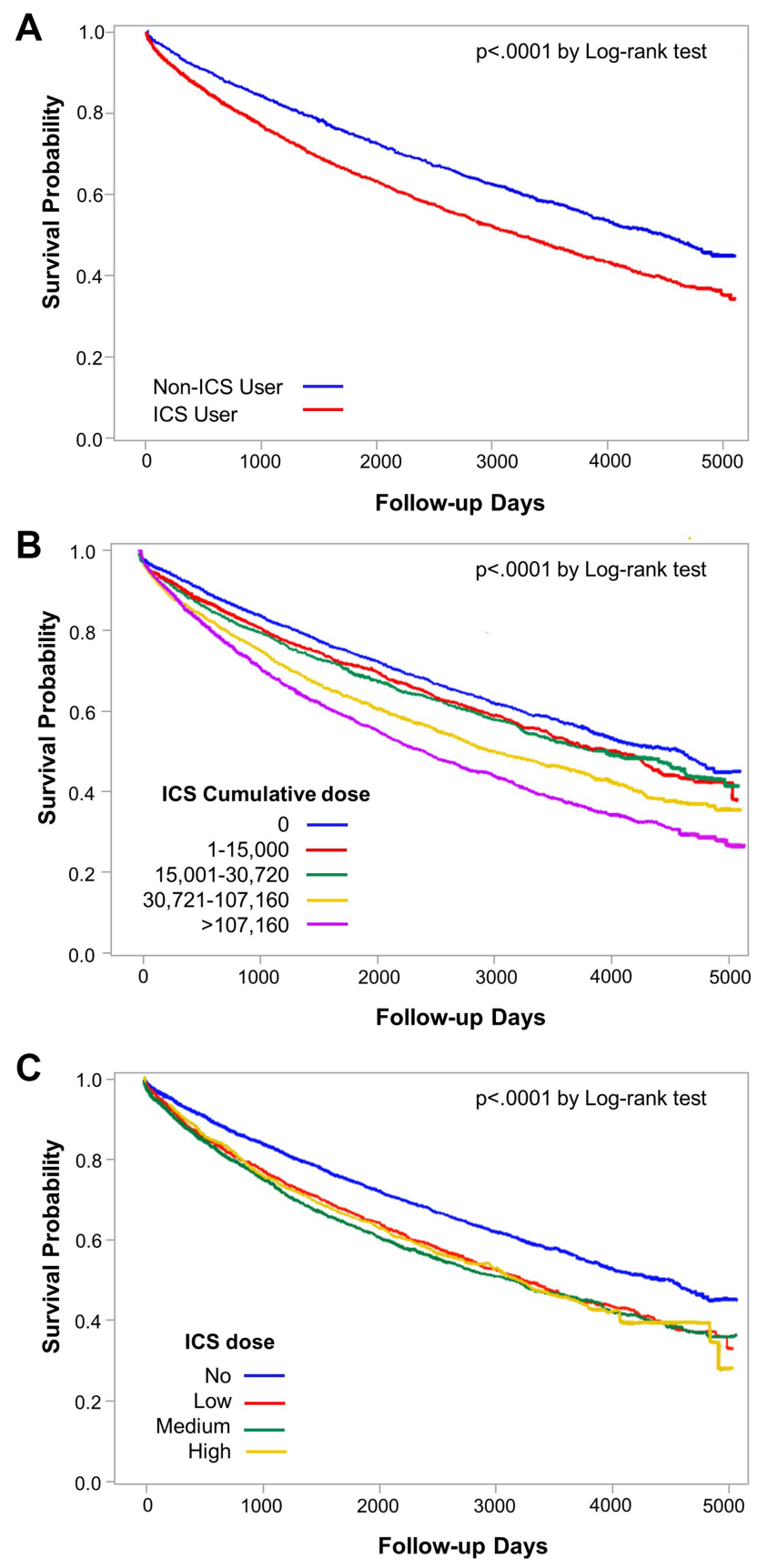

Figure 2 Survival analysis of the development of pneumonia using Kaplan-Meier curve according to the use of inhaled corticosteroid (ICS) (A), cumulative dose of ICS (B), and daily dose of ICS (C).

$(\mathrm{BMI})$ is an independent risk factor of pneumonia in COPD. $^{27}$ The mean BMI of patients enrolled in the Korean COPD cohort was $23 \pm 3 \mathrm{~kg} / \mathrm{m}^{2}$, which is lower than $27 \pm$ $6 \mathrm{~kg} / \mathrm{m}^{2}$ observed in the Western COPD cohort in the Evaluation of COPD Longitudinally to Identify Predictive Surrogate Endpoints (ECLIPSE) study. ${ }^{28}$ Another characteristic was lung function. Lung function, such as $\mathrm{FEV}_{1}$ and FVC, is usually presented as a predictive value (\%). However, the absolute value of lung function from healthy adults presented in liters was significantly lower in Asians than in whites. ${ }^{29}$ In this regard, although COPD patients from different races have the same airflow limitations determined via predictive values, their absolute lung functions might be significantly different. Lower lung function was found to be an independent risk factor for pneumonia, and the recommended dosage of ICS is consistent regardless of ethnic differences. $^{5,27}$ Therefore, the risk of pneumonia could be increased in the Korean population. The present study had 


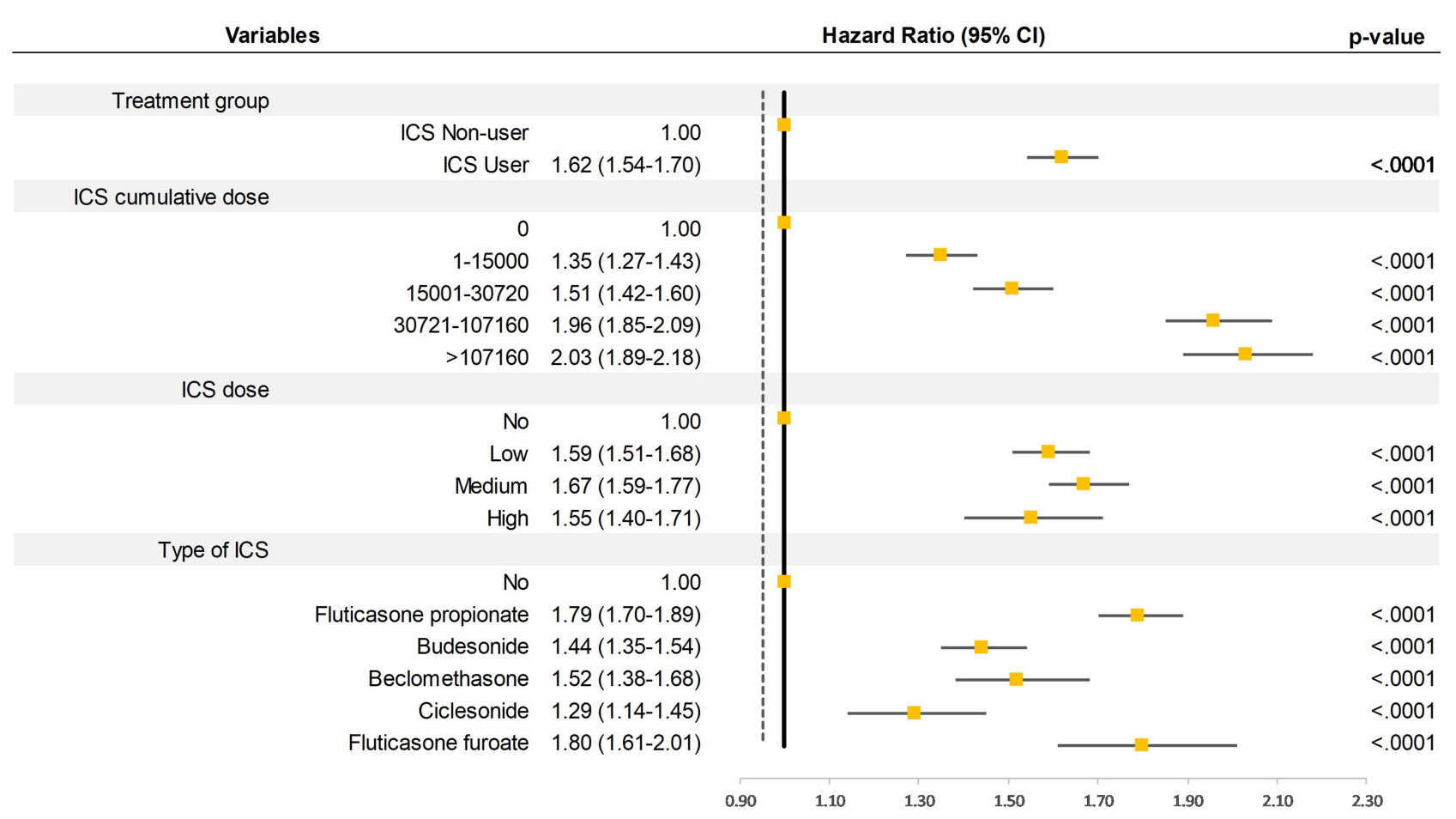

Figure 3 Hazard ratios of pneumonia according to the use of inhaled corticosteroid (ICS) and subtype of ICS users.

a long observational period of 13 years, while the observational period of other studies was usually 5 years. ${ }^{20}$ Therefore, differences in the incidence of pneumonia between ICS users and non-ICS users might become prominent during longer observation periods.

Cumulative doses of ICSs were rarely taken into account when considering the risk of pneumonia. Instead, the daily doses of ICS, classified into low, medium and high doses, were regarded as significant factors associated with pneumonia. An observational study examining the commercial healthcare database of an American population reported that the HR of pneumonia increased as the daily ICS doses increased: 1.38 (1.27-1.49), 1.69 (1.52-1.88), and 2.57 (1.98-3.33) for low, medium, and high doses of ICS, respectively. ${ }^{25}$ Another observational study analyzing Taiwan's nationwide, populationbased database also showed that the odds ratio of pneumonia increased with an increment in the daily ICS dose: 1.10 (1.04-1.16), 1.33 (1.26-1.39), and 1.63 (1.50-1.78) for low, medium, and high doses of ICS, respectively. ${ }^{26}$ Side effects from the cumulative dose of ICS in COPD patients, particularly elderly patients, include tuberculosis, risk of fractures, and cataracts. ${ }^{13,30}$ The reason for the link between the risk of pneumonia and the cumulative dose of ICS in the present study seems inexplicable. Although ICS use is an effective treatment strategy, its overuse has been increasingly recognized. Almost $70 \%$ of COPD patients were reported to receive
ICSs when enrolled in RCTs and market surveys. ${ }^{31}$ In the present Korea NHIS data, 59,694/87,594 (68.15\%) COPD patients were treated with ICS. Therefore, ICSs may be overly prescribed in the actual clinical setting for the treatment of COPD. Given that the cumulative dose of ICS is another risk factor for pneumonia, we need to follow the recommendation of COPD guidelines that recommend that ICSs should be discontinued if pneumonia or a lack of response to ICSs is present. ${ }^{5}$ In the observational study by Suissa., et al, the risk of pneumonia gradually decreased after discontinuation of ICS and disappeared after 6 months. ${ }^{21}$

It remains to be confirmed whether some types of ICS result in a higher risk of pneumonia, since most study results were derived from indirect comparisons between fluticasone propionate and budesonide in the meta-analyses and observational studies. In the meta-analyses, fluticasone propionate was consistently associated with an increased risk of pneumonia (odds ratio, 1.43-1.75), while budesonide was not associated with pneumonia (odds ratio of $0.84-1.19$ ).${ }^{32-36}$ In the observational study by Suissa et al, the relative risk of pneumonia was $2.01(1.93-2.10)$ for fluticasone propionate compared to 1.17 (1.09-1.26) for budesonide. ${ }^{21}$ In the study using Swedish registry medical records, the rate ratio of pneumonia in subjects treated with fluticasone propionate in comparison to those treated with budesonide was $1.73(1.57-1.90) .{ }^{37}$ A Taiwan's population-based study showed a significantly increased risk 
of pneumonia in subjects treated with a fluticasone propionatecontaining regimen (odds ratio, 1.22-1.35), but no statistically significant risk of pneumonia in those treated with a budesonide-containing regimen. ${ }^{26}$ The present study results are consistent with those of previous studies that a higher risk of pneumonia was associated with fluticasone propionate use than with budesonide (HR, 1.79 (1.70-1.89) versus 1.44 (1.$35-1.54)$ ). In addition, fluticasone furoate was highly associated with pneumonia (1.80 (1.61-2.01)). Therefore, the type of ICS should be carefully determined when prescribing ICSs to patients with risk factors for pneumonia, and subsequent observational studies are needed to confirm the different risks of pneumonia according to ICS subtypes.

The strength of the present study is its study design: we performed a nationwide population-based cohort study with a long-term observational period. Almost all Korean populations were included in the NHIS so that it could be the representative data of our country. While other observational studies adopted a case-control design, ${ }^{21-24}$ the present study was conducted by using a cohort design to clarify the causal relationship between ICS use and the risk of pneumonia. In addition, daily doses of ICS and some type of ICS, such as fluticasone propionate and budesonide, were mainly reported as risk factors for pneumonia in other observational studies. ${ }^{20}$ However, the cumulative dose of the ICS and every type of ICS prescribed for COPD in realworld clinical settings were compared in the present study.

There are limitations to this study. First, although we performed 1:1 propensity score matching, the severity of COPD might not match for ICS users and non-ICS users without the available clinical information of COPD in the claims database. Instead, COPD admission, OCS prescription, and OCS prescription duration were used as surrogates for COPD severity in the propensity score matching. Second, diagnosis of pneumonia was only based on diagnostic codes and was not radiologically confirmed. However, this is an inherent issue in most studies related to pneumonia, regardless of the study design. Third, the proportion of asthma as a comorbidity was approximately $50 \%$ in both groups. Although some proportion of subjects with asthma as a comorbidity had asthma-COPD overlap features, this might be due to the definition of comorbidities, which was solely based on the diagnostic codes. Because at least two diagnostic codes were recorded during the entire study period, these were defined as comorbidities. A population-based study from Taiwan, which has a healthcare system similar to Korea, also reported a high proportion of asthma as a comorbidity (approximately 50\%). ${ }^{26}$

\section{Conclusion}

The use of ICS increases the risk of pneumonia in patients with COPD. Therefore, ICSs should be carefully prescribed in patients with risk factors for pneumonia, considering the cumulative doses and subtypes of ICS.

\section{Acknowledgment}

This study has not been presented elsewhere.

\section{Funding}

There was no funding for this research.

\section{Disclosure}

The authors report no conflicts of interest in this work.

\section{References}

1. Riley CM, Sciurba FC. Diagnosis and outpatient management of chronic obstructive pulmonary disease: a review. JAMA. 2019;321 (8):786-797. doi:10.1001/jama.2019.0131

2. Deas SD, Huprikar N. Dual bronchodilator therapy for chronic obstructive pulmonary disease: evidence for the efficacy and safety of fixed dose combination treatments in the setting of recent guideline updates. Curr Opin Pulm Med. 2018;24(2):130-137. doi:10.1097/ MCP.0000000000000450

3. Tashkin DP, Strange C. Inhaled corticosteroids for chronic obstructive pulmonary disease: what is their role in therapy? Int J Chron Obstruct Pulmon Dis. 2018;13:2587-2601. doi:10.2147/COPD.S172240

4. Agusti A, Fabbri LM, Singh D, et al. Inhaled corticosteroids in COPD: friend or foe? Eur Respir J. 2018;52(6):1801219. doi:10.1183/13993003.01219-2018

5. Global initiative for chronic lung disease (GOLD): global strategy for the diagnosis, management, and prevention of COPD; 2020. Available from: www.globalcopd.org. Accessed August 22, 2020.

6. Avdeev S, Aisanov Z, Arkhipov V, et al. Withdrawal of inhaled corticosteroids in COPD patients: rationale and algorithms. Int J Chron Obstruct Pulmon Dis. 2019;14:1267-1280. doi:10.2147/COPD.S207775

7. Williams DM. Clinical pharmacology of corticosteroids. Respir Care. 2018;63(6):655-670. doi:10.4187/respcare.06314

8. Tashkin DP, Miravitlles M, Celli BR, et al. Concomitant inhaled corticosteroid use and the risk of pneumonia in COPD: a matched-subgroup post hoc analysis of the UPLIFT(R) trial. Respir Res. 2018;19(1):196. doi:10.1186/s12931-018-0874-0

9. Kew KM, Seniukovich A. Inhaled steroids and risk of pneumonia for chronic obstructive pulmonary disease. Cochrane Database Syst Rev. 2014;3(3):Cd010115. doi:10.1002/14651858.CD010115.pub2

10. Hirano R, Fujita M, Matsumoto T, On R, Watanabe K. Inhaled corticosteroids might not increase the risk of pneumonia in patients with chronic obstructive pulmonary disease in Japan. Int J Chron Obstruct Pulmon Dis. 2018;13:3503-3509. doi:10.2147/COPD.S180349

11. Price D, Brusselle G, Roche N, Freeman D, Chisholm A. Real-world research and its importance in respiratory medicine. Breathe (Sheff). 2015;11(1):26-38. doi:10.1183/20734735.015414

12. Cho KH, Kim YS, Linton JA, Nam CM, Choi Y, Park EC. Effects of inhaled corticosteroids/long-acting agonists in a single inhaler versus inhaled corticosteroids alone on all-cause mortality, pneumonia, and fracture in chronic obstructive pulmonary disease: a nationwide cohort study 2002-2013. Respir Med. 2017;130:75-84. doi:10.1016/ j.rmed.2017.07.012 
13. Lee CH, Kim K, Hyun MK, Jang EJ, Lee NR, Yim JJ. Use of inhaled corticosteroids and the risk of tuberculosis. Thorax. 2013;68 (12):1105-1113. doi:10.1136/thoraxjnl-2012-203175

14. Calverley PM, Anderson JA, Celli B, et al. Salmeterol and fluticasone propionate and survival in chronic obstructive pulmonary disease. $N$ Engl J Med. 2007;356(8):775-789. doi:10.1056/NEJMoa063070

15. Calverley PMA, Stockley RA, Seemungal TAR, et al. Reported pneumonia in patients with COPD: findings from the INSPIRE study. Chest. 2011;139(3):505-512. doi:10.1378/chest.09-2992

16. Singh S, Loke YK. Risk of pneumonia associated with long-term use of inhaled corticosteroids in chronic obstructive pulmonary disease: a critical review and update. Curr Opin Pulm Med. 2010;16 (2):118-122. doi:10.1097/MCP.0b013e328334c085

17. Tashkin DP, Rennard SI, Martin P, et al. Efficacy and safety of budesonide and formoterol in one pressurized metered-dose inhaler in patients with moderate to very severe chronic obstructive pulmonary disease: results of a 6-month randomized clinical trial. Drugs. 2008;68(14):1975-2000. doi:10.2165/00003495-200868140-00004

18. Rennard SI, Tashkin DP, McElhattan J, et al. Efficacy and tolerability of budesonide/formoterol in one hydrofluoroalkane pressurized metered-dose inhaler in patients with chronic obstructive pulmonary disease: results from a 1-year randomized controlled clinical trial. Drugs. 2009;69(5):549-565. doi:10.2165/00003495-200969050-00004

19. Welte T, Miravitlles M, Hernandez P, et al. Efficacy and tolerability of budesonide/formoterol added to tiotropium in patients with chronic obstructive pulmonary disease. Am J Respir Crit Care Med. 2009;180 (8):741-750. doi:10.1164/rccm.200904-0492OC

20. Finney L, Berry M, Singanayagam A, Elkin SL, Johnston SL, Mallia P. Inhaled corticosteroids and pneumonia in chronic obstructive pulmonary disease. Lancet Respir Med. 2014;2(11):919-932. doi:10.1016/s2213-2600(14)70169-9

21. Suissa S, Patenaude V, Lapi F, Ernst P. Inhaled corticosteroids in COPD and the risk of serious pneumonia. Thorax. 2013;68 (11):1029-1036. doi:10.1136/thoraxjnl-2012-202872

22. Ernst P, Gonzalez AV, Brassard P, Suissa S. Inhaled corticosteroid use in chronic obstructive pulmonary disease and the risk of hospitalization for pneumonia. Am J Respir Crit Care Med. 2007;176 (2):162-166. doi:10.1164/rccm.200611-1630OC

23. Thornton Snider J, Luna Y, Wong KS, et al. Inhaled corticosteroids and the risk of pneumonia in medicare patients with COPD. Curr Med Res Opin. 2012;28(12):1959-1967. doi:10.1185/03007995.2012.743459

24. Joo MJ, Au DH, Fitzgibbon ML, Lee TA. Inhaled corticosteroids and risk of pneumonia in newly diagnosed COPD. Respir Med. 2010;104 (2):246-252. doi:10.1016/j.rmed.2009.10.002

25. Yawn BP, Li Y, Tian H, Zhang J, Arcona S, Kahler KH. Inhaled corticosteroid use in patients with chronic obstructive pulmonary disease and the risk of pneumonia: a retrospective claims data analysis. Int J Chron Obstruct Pulmon Dis. 2013;8:295-304. doi:10.2147/COPD.S42366

26. Wang CY, Lai CC, Yang WC, et al. The association between inhaled corticosteroid and pneumonia in COPD patients: the improvement of patients' life quality with COPD in Taiwan (IMPACT) study. Int J Chron Obstruct Pulmon Dis. 2016;11:2775-2783. doi:10.2147/ COPD.S116750
27. Crim C, Dransfield MT, Bourbeau J, et al. Pneumonia risk with inhaled fluticasone furoate and vilanterol compared with vilanterol alone in patients with COPD. Ann Am Thorac Soc. 2015;12(1):27-34. doi:10.1513/AnnalsATS.201409-413OC

28. Lim JU, Lee JH, Kim JS, et al. Comparison of World Health Organization and asia-pacific body mass index classifications in COPD patients. Int $J$ Chron Obstruct Pulmon Dis. 2017;12:2465-2475. doi:10.2147/COPD.S141295

29. Hankinson JL, Kawut SM, Shahar E, Smith LJ, Stukovsky KH, Barr RG. Performance of American thoracic society-recommended spirometry reference values in a multiethnic sample of adults: the multi-ethnic study of atherosclerosis (MESA) lung study. Chest. 2010;137(1):138-145. doi:10.1378/chest.09-0919

30. Battaglia S, Cardillo I, Lavorini F, Spatafora M, Scichilone N. Safety considerations of inhaled corticosteroids in the elderly. Drugs Aging. 2014;31(11):787-796. doi:10.1007/s40266-014-0213-1

31. Cataldo D, Derom E, Liistro G, et al. Overuse of inhaled corticosteroids in COPD: five questions for withdrawal in daily practice. Int J Chron Obstruct Pulmon Dis. 2018;13:2089-2099. doi:10. 2147/COPD.S164259

32. Halpin DM, Gray J, Edwards SJ, Morais J, Singh D. Budesonide/ formoterol vs. salmeterol/fluticasone in COPD: a systematic review and adjusted indirect comparison of pneumonia in randomised controlled trials. Int J Clin Pract. 2011;65(7):764-774. doi:10.1111/ j.1742-1241.2011.02685.x

33. Nannini LJ, Lasserson TJ, Poole P. Combined corticosteroid and long-acting beta(2)-agonist in one inhaler versus long-acting beta (2)-agonists for chronic obstructive pulmonary disease. Cochrane Database Syst Rev. 2012;9(9):Cd006829. doi:10.1002/14651858. CD006829.pub2

34. Nannini LJ, Poole P, Milan SJ, Holmes R, Normansell R. Combined corticosteroid and long-acting beta2-agonist in one inhaler versus placebo for chronic obstructive pulmonary disease. Cochrane Database Syst Rev. 2013;11(11):Cd003794. doi:10.1002/14651858. CD003794.pub4

35. Spencer S, Karner C, Cates CJ, Evans DJ. Inhaled corticosteroids versus long-acting beta(2)-agonists for chronic obstructive pulmonary disease. Cochrane Database Syst Rev. 2011;12(12):Cd007033. doi:10.1002/14651858.CD007033.pub3

36. Janson C, Stratelis G, Miller-Larsson A, Harrison TW, Larsson K. Scientific rationale for the possible inhaled corticosteroid intraclass difference in the risk of pneumonia in COPD. Int J Chron Obstruct Pulmon Dis. 2017;12:3055-3064. doi:10.2147/COPD.S143656

37. Janson C, Larsson K, Lisspers KH, et al. Pneumonia and pneumonia related mortality in patients with COPD treated with fixed combinations of inhaled corticosteroid and long acting beta2 agonist: observational matched cohort study (PATHOS). BMJ. 2013;346:f3306. doi:10.1136/bmj.f3306

\section{Publish your work in this journal}

The International Journal of COPD is an international, peer-reviewed journal of therapeutics and pharmacology focusing on concise rapid reporting of clinical studies and reviews in COPD. Special focus is given to the pathophysiological processes underlying the disease, intervention programs, patient focused education, and self management protocols. This journal is indexed on PubMed Central, MedLine and CAS. The manuscript management system is completely online and includes a very quick and fair peer-review system, which is all easy to use. Visit http://www.dovepress.com/testimonials.php to read real quotes from published authors. 